\title{
The SH-SY5Y cell line in Parkinson's disease research: a systematic review
}

\author{
Helena Xicoy ${ }^{1,2}$, Bé Wieringa ${ }^{1}$ and Gerard J.M. Martens ${ }^{2^{*}}$
}

\begin{abstract}
Parkinson's disease (PD) is a devastating and highly prevalent neurodegenerative disease for which only symptomatic treatment is available. In order to develop a truly effective disease-modifying therapy, improvement of our current understanding of the molecular and cellular mechanisms underlying PD pathogenesis and progression is crucial. For this purpose, standardization of research protocols and disease models is necessary. As human dopaminergic neurons, the cells mainly affected in PD, are difficult to obtain and maintain as primary cells, current PD research is mostly performed with permanently established neuronal cell models, in particular the neuroblastoma SH-SY5Y lineage. This cell line is frequently chosen because of its human origin, catecholaminergic (though not strictly dopaminergic) neuronal properties, and ease of maintenance. However, there is no consensus on many fundamental aspects that are associated with its use, such as the effects of culture media composition and of variations in differentiation protocols. Here we present the outcome of a systematic review of scientific articles that have used SH-SY5Y cells to explore PD. We describe the cell source, culture conditions, differentiation protocols, methods/approaches used to mimic PD and the preclinical validation of the SH-SY5Y findings by employing alternative cellular and animal models. Thus, this overview may help to standardize the use of the SH-SY5Y cell line in PD research and serve as a future user's guide.
\end{abstract}

Keywords: Cell culture conditions, Cellular differentiation, Cellular model, Dopaminergic neuron, Neuroblastoma, Parkinson's disease, SH-SY5Y cell line

\section{Background}

Parkinson's disease (PD) is the second most common neurodegenerative disease with a predicted prevalence of 9 million people worldwide by 2030 [1, 2]. PD has a high socioeconomic burden since it is slowly progressive and disease-modifying treatments are not available. PD presents with motor and non-motor symptoms [3, 4] that worsen with advancing age, leading to a need for assistance with all daily activities. Disease manifestation is characterized by the presence of Lewy bodies (abnormal protein aggregates containing $\alpha$-synuclein), death of dopaminergic (DAergic) neurons in the substantia nigra (SN) projecting to the striatum, and microgliosis (accumulation of activated microglial cells) [5]. However, the molecular mechanisms underlying all these disease features are unknown, hampering the development of effective treatment. In order to understand the

\footnotetext{
* Correspondence: G.Martens@ncmls.ru.nl

${ }^{2}$ Department of Molecular Animal Physiology, Donders Institute for Brain, Cognition and Behaviour, Radboud University, Nijmegen, The Netherlands Full list of author information is available at the end of the article
}

pathophysiological mechanisms underlying PD and develop disease-modifying therapies, it is necessary to have adequate models for in vitro and in vivo studies.

An in vitro model widely used in PD research is the neuroblastoma SH-SY5Y cell line. This line is a subline of the SK-N-SH cell line, which was established in culture in 1970 from a bone marrow biopsy of a metastatic neuroblastoma of a 4-year-old female and has undergone three rounds of clonal selection [6]. The initial characterization of the SH-SY5Y cell line showed moderate activity of dopamine- $\beta$-hydroxylase and negligible levels of choline acetyl-transferase, acetylcholinesterase and butyryl-cholinesterase [6], basal noradrenaline (NA) release [7] and tyrosine hydroxylase activity [8]. Tyrosine hydroxylase is the rate-limiting enzyme of the catecholamine synthesis pathway and converts tyrosine to L-dopa [9], the precursor of dopamine (DA), which is converted to NA by dopamine- $\beta$ hydroxylase [10]. Therefore, the SH-SY5Y cell line may display a catecholaminergic phenotype since it has the machinery to synthesize both DA and NA. Although 
these properties do not classify SH-SY5Y cells as purely DAergic, this cell line has been widely used as a model for PD. The SH-SY5Y cell line displays a number of genetic aberrations due to its cancerous origin, but most genes and pathways dysregulated in PD pathogenesis are intact [11]. However, the use of an oncogenically transformed cell line with catecholaminergic rather than exclusively DAergic properties remains a controversial issue in the PD field.

The purpose of this systematic review is to provide an overview of the value and use of the SH-SY5Y line as a cell model for PD. We describe in detail the culture conditions, the methodology used to provoke the onset of differentiation, the techniques to mimic typical pathogenic PD features and the alternative models used to validate the findings. Moreover, the limitations of the use of the cell line will be discussed.

\section{Main text}

For our review, we conducted a standard systematic literature search in PubMed which included the terms "Parkinson", "Parkinson's", or "Parkinson's disease" and "neuroblastoma", "SH-SY5Y", "SHSY5Y" or "SHSY-5Y". The application of these search terms aimed to cover most of the literature regarding the use of the SH-SY5Y cell line in PD research, missing only those studies in which the above-mentioned terms are only present in the main text but not in the title, abstract or $\mathrm{MeSH}$ terms. The search performed the $22^{\text {nd }}$ of November 2016 retrieved 1489 articles, of which 962 were original, accessible and PD-specific papers and thus were included in the analysis. The exclusion criteria for the remaining 527 articles were: (i) written in a language different from English, (ii) represents a review, (iii) not specific for PD, (iv) Parkinson as an author, (v) the cell line was mentioned but used in previous studies, and (vi) use of a neuroblastoma cell line different from SH-SY5Y (see Additional file 1, for full list and exclusion details).

\section{Cell source and culture conditions}

The most-reported source for access to the SH-SY5Y cell line is the American Type Culture Collection (ATCC, CRL-2266, deposited by JL Biedler). Other sources concern retrieval from other cell banks, such as the European Collection of Authenticated Cell Cultures (ECACC, Catalog number: 94030304, deposited by PFT Vaughan) or the German collection of Microorganisms and Cell Cultures (DSMZ, ACC 209). Cells were also obtained through gifts from colleague scientists (Fig. 1). However, in 455 out of 962 publications the cell origin was not specified.

Recommendations regarding the composition of the growth medium for propagation of SH-SY5Y cells vary among the various cell line distributors: ATCC

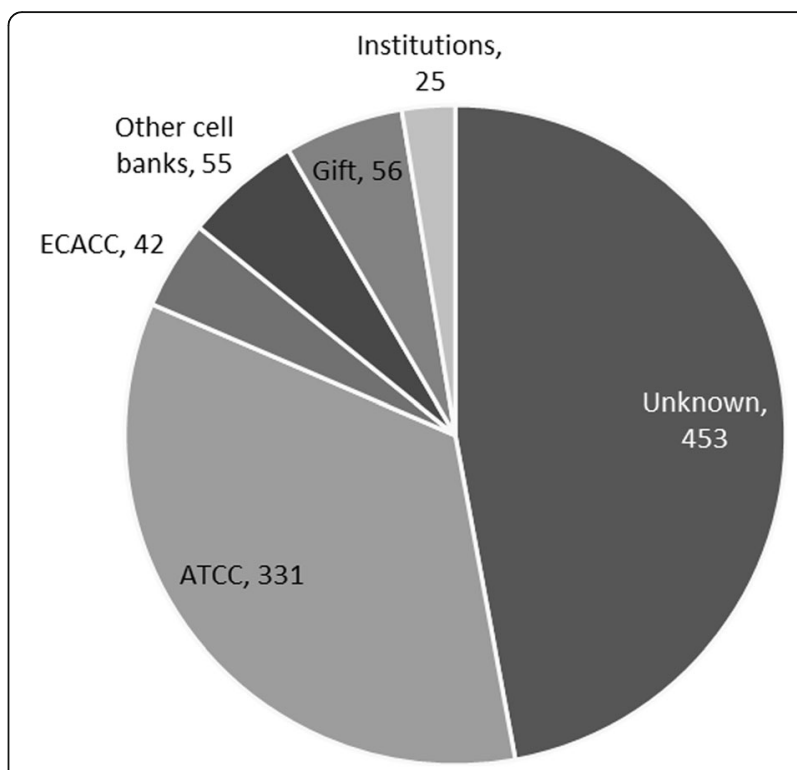

Fig. 1 Sources from which researchers obtained the SH-SY5Y cell line. Number of articles using a particular source is indicated. ATCC: American Type Culture Collection; EACC: European Collection of Authenticated Cell Cultures; other cell banks includes all of them, except for ATCC and ECACC

recommends MEM/F12 supplemented with 10\% fetal bovine serum, ECCAC recommends MEM/F12 with $2 \mathrm{mM}$ glutamine, 1\% non-essential amino acids and 15\% fetal bovine serum, and DSMZ recommends MEM plus $15-20 \%$ fetal bovine serum. In the actual protocols employed in the PD-related publications, DMEM was used most (434 out of 962 publications), followed by DMEM/F12 (230 out of 962 publications), MEM/F12 (68 out of 962 publications), DMEM high glucose (46 out of 962 publications), RPMI 1640 (37 out of 962 publications), Cosmedium-001 (21 out of 962 publications) and MEM (20 out of 962 publications) (Table 1). Furthermore, media used were supplemented with antibiotics/antimycotics $(65.6 \%$ of the articles), glutamine (23.9\% of the articles), non-essential amino acids $(9.8 \%$ of the articles), sodium pyruvate (6.3\% of the articles) or other components, such as HEPES, sodium carbonate, uridine or L-lysine, in different combinations (Table 1; for more detailed information of the media composition of each article, see Additional file 2). Careful choice of medium type and composition is crucial, e.g. the use of DMEM or RPMI changed the metabolome and the differentiation capacity of a number of cell lines [12, 13]. Evidence is now accumulating that nutrient availability, and also the degree of oxygenation, affects wiring through different metabolic pathways and that the intracellular levels of glutamine, alpha-ketoglutarate, pyruvate and $\mathrm{NAD}+/ \mathrm{NADH}$ redox ratio and concentration are determining factors in the epigenetic control of gene expression during differentiation, e.g. via effects on 
Table 1 Compositions of the media used for culturing SH-SY5Y cells

\begin{tabular}{|c|c|c|c|c|c|}
\hline \multicolumn{2}{|l|}{ Basal media } & \multicolumn{2}{|c|}{ Supplements - serum } & \multicolumn{2}{|l|}{ Supplements - others } \\
\hline Name & \#articles & Name & \#articles & Name & \#articles \\
\hline DMEM & 434 & $10 \%$ FBS & 770 & Antibiotics/antimycotics & 631 \\
\hline DMEM/F12 & 230 & $15 \%$ FBS & 70 & & \\
\hline MEM/F12 & 68 & $5 \%$ FBS & 33 & Glutamine/GlutaMAX & 230 \\
\hline DMEM (high glucose) & 46 & $20 \%$ FBS & 3 & & \\
\hline RPMI 1640 & 37 & None & 2 & NEAA & 94 \\
\hline Cosmedium-001 & 21 & Others & 14 & Sodium pyruvate & 61 \\
\hline MEM & 20 & Unknown & 70 & & \\
\hline Other & 36 & & & & \\
\hline Unknown & 70 & & & & \\
\hline
\end{tabular}

The table is divided into three parts (basal media, serum supplement and other supplements) and the number of papers involved is indicated (\#articles). The Additional file 2 contains a more detailed description of all media and supplements used. Unknown refers to the articles that do not specify media composition. DMEM: Dulbecco's Modified Eagle Medium; F12: nutrient mixture F12, MEM: Minimum Essential Media; FBS: fetal bovine serum; NEAA: non-essential amino acids

histone-lysine demethylases and DNA demethylases [14]. Furthermore, supplementing media with sodium pyruvate has been shown to be protective against oxidative stress $[15,16]$ and as such may affect the outcome of experiments that study the involvement of oxidative damage in PD. In $80 \%$ of the articles, the media described were supplemented with $10 \%$ fetal bovine serum, although some authors use other concentrations of serum, ranging from 5 to $20 \%$, or serum from other species, such as horse (Table 1). Taking into account that serum includes growth factors, hormones, amino acids and lipids that can influence cell growth and differentiation, the use of different serum concentrations, serum from different species or even serum from different batches may influence the outcome [17]. It is of note here that variability in cell growth and sensitivity to various compounds depending on the media and substrate used for the growth of SH-SY5Y cells has already been reported [18].

To appropriately acknowledge the various effects that differences in culture media composition can have on the cellular phenotype, it is thus imperative to systematically identify in future studies how various metabolic intermediates, ions, serum constituents and substrates influence aspects of growth and differentiation of $\mathrm{SH}$ SY5Y cells. Only then the protocols for experiments with PD cell models can be widely standardized.

\section{Phenotype and differentiation of SH-SY5Y cells}

The use of the SH-SY5Y cell line is not restricted to PDresearch; this cell line has also been used in other areas of neuroscience, including research on Alzheimer's disease, neurotoxicity, ischemia or Amyotrophic Lateral Sclerosis, among others $[11,19,20]$. To obtain derivative cells with a neuronal phenotype, multiple differentiation protocols have been described [21-25], but details about the final population of cells, regarding the fate-choices and fate-specification of cells that undergo terminal differentiation, have not been systematically reported. Since PD is characterized by the death of SN DAergic neurons, the degree to which this cell line displays a DAergic phenotype is a key aspect regarding the validity of the model. In this respect, 392 out of the 962 papers state that SH-SY5Y cells have a DAergic phenotype without actually showing supporting evidence. Only a few papers cite previous work showing the DAergic phenotype. Another large proportion of articles (432 out of 962) does not provide any statement about the DAergic phenotype or the rationale behind the choice of the cell line for use in PD-research. Among the remaining publications, in 76 papers the SH-SY5Y lineage is represented as a PDmodel with DAergic properties or a toxin-induced PD-like phenotype, another 56 publications report the analysis of the DAergic phenotype, and in 7 papers cholinergic, neuronal or noradrenergic phenotypes are mentioned (Table 2).

The phenotype of SH-SY5Y cells can be manipulated by inducing different programs of terminal neural differentiation. However, in $81,5 \%$ of the published studies no differentiation regime was used (Fig. 2), for which in only seven publications a reason was given. Among the studies that do report on forced differentiation, the most common method employed is the addition of retinoic acid (RA) in concentrations ranging from $5 \mu \mathrm{M}$ to $100 \mu \mathrm{M}$, for a period of time from 24 hours to 21 days, and, sometimes, a reduction of the concentration of serum in the media (Fig. 2). It has been reported that RA treatment upregulates expression of neuronal and DAergic markers and increases susceptibility to DAergic neurotoxins [26]. However, other studies have observed increased neuronal markers upon RA differentiation, but no change in DAergic markers and decreased susceptibility to DAergic neurotoxins [27]. The phenotypic effect of RA on SH-SY5Y cells has been systematically studied, 
Table 2 Papers reporting the DAergic phenotype of the SHSY5Y cell line and techniques used

\begin{tabular}{lll}
\hline DAergic phenotype & \#articles & \#differentiated \\
\hline Not stated & 432 & 70 \\
Stated and not checked & 392 & 50 \\
PD model/DAergic properties/toxin & 76 & 12 \\
Stated and checked $^{\text {a }}$ & 48 & 22 \\
Not stated but checked $^{a}$ & 7 & 3 \\
Others & 7 & 2 \\
Technique & Single & Multiple \\
ICC & $11(4 / 7)$ & $15(6 / 9)$ \\
WB & $10(6 / 4)$ & $23(13 / 10)$ \\
qPCR & $1(1 / 0)$ & $13(7 / 6)$ \\
DA uptake/content & $2(2 / 0)$ & $6(3 / 3)$ \\
Not shown & $4(3 / 1)$ & 0 \\
\hline
\end{tabular}

The top part of the table indicates the number of papers that do specify or do not specify the DAergic phenotype of the SH-SY5Y cell line and whether or not the authors checked the phenotype. "Others" refers to articles mentioning other neuronal linages, including cholinergic, neuronal and noradrenergic phenotypes. Shown are the total number of articles (\#articles) and the number of articles in which forced differentiation was employed (\#differentiated). The bottom part of the table summarizes the techniques used in the publications that checked the phenotype ( $\left.{ }^{\mathrm{a}}\right)$. Publications are divided into 'single' (i.e. papers that use only one method to check the DAergic phenotype) and 'multiple' (papers that use multiple complementary techniques). Between brackets: the number of studies that checked the DAergic phenotype in undifferentiated/differentiated cells. ICC immunocytochemistry, WB western blot, $q P C R$ quantitative polymerase chain reaction, $D A$ dopamine

including the induction of a terminal neural phenotype with, specifically, a DAergic-like character [28]. Conversely, RA-mediated differentiation of SH-SY5Y cells has been associated with the induction of a cholinergic rather than DAergic phenotype [29]. Here it is important to note that RA has been found to partially protect $\mathrm{SH}-\mathrm{SY} 5 \mathrm{Y}$ cells against proteasome inhibitors [30]. In view of this finding, the results of studies examining proteasomal dysfunction and involving RA-differentiated SH-SY5Y cells as PD-model should be interpreted with care. The second method of choice to differentiate SHSY5Y cells is a sequential treatment with RA, usually $10 \mu \mathrm{M}$, and 12-O-Tetradecanoylphorbol-13-acetate (TPA), mostly added in a concentration of 80nM (Fig. 2). This protocol has been demonstrated to differentiate SH-SY5Y cells more efficiently to DAergic-like neurons [31-33]. Early studies on the use of RA and TPA (alone or in combination) to differentiate SH-SY5Y cells have shown that these compounds induce various neuronallike populations, with a strong increase of NA content when using only TPA [7]. In view of these differences, it is important to realize that a set of neurons each synthesizing a separate neurotransmitter (s) has a distinct transcriptional profile [34]. Even neurons synthesizing a specific neurotransmitter can be classified into several subpopulations, each with a clearly defined signaling function in a particular (brain) region and an explicit vulnerability for stress factors [35]. The third approach that is commonly used for differentiation induction involves the sequential treatment with RA, usually $10 \mu \mathrm{M}$, and $10-100 \mathrm{ng} / \mathrm{mL}$ of brain-derived neurotrophic factor (BDNF) (Fig. 2). This procedure leads to a homogeneous neuronal population with expression of neuronal markers and decreased proliferation [21]. The phenotypic outcome of this RA/BDNF differentiation protocol is, however, still somewhat controversial as it has been described as sympathetic cholinergic, based on evidence from target-directed qPCR and microarray studies which pointed into the direction of increased levels of acetylcholine transporter, choline acetyl transferase and neuropeptide $\mathrm{Y}[36,37]$, but also as dopaminergic by others [38]. Moreover, inhibition of cell growth has not always been replicated when employing this procedure [24].

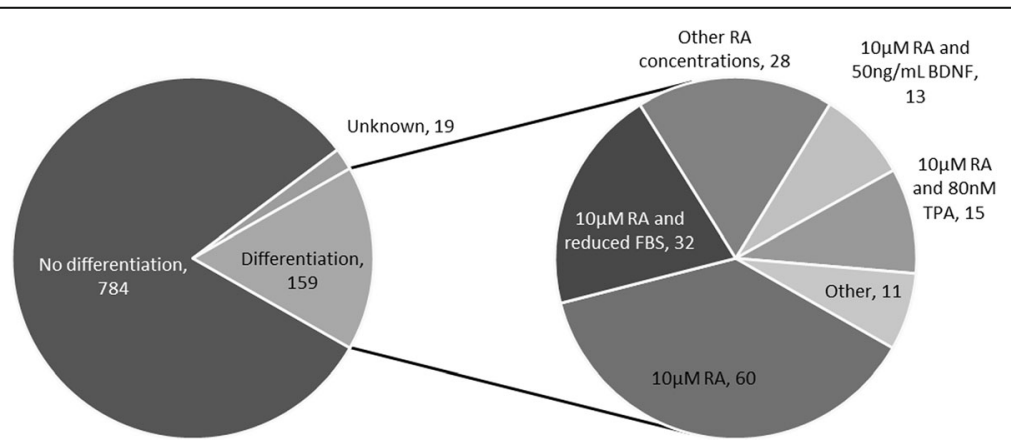

Fig. 2 Papers reporting the differentiation of the SH-SY5Y cell line for PD-research. Left: Proportion of studies that do not use differentiation protocols (no differentiation), those that do not specify the differentiation status (unknown) and those that include a differentiation regime (differentiation). Among the papers in which differentiated cells were used, the main differentiation treatments used are depicted in the right chart, including $10 \mu \mathrm{M}$ retinoic acid (RA), $10 \mu \mathrm{M}$ RA and reduced fetal bovine serum (FBS), other concentrations of RA, $10 \mu \mathrm{M}$ RA and $50 \mathrm{ng} / \mathrm{ml}$ brain-derived neurotrophic factor (BDNF) and $10 \mu \mathrm{M}$ RA and 80nM 12-O-Tetradecanoylphorbol-13-acetate (TPA). Other includes $10 \mu \mathrm{M}$ RA, 1\%FBS and 0.3 mM dibutyryl-cAMP; $10 \mu \mathrm{M}$ RA or $10 \mu \mathrm{g} / \mathrm{mL}$ BDNF; $10 \mu \mathrm{M}$ RA and 80nM TPA or $50 \mathrm{ng} / \mathrm{mL}$ BDNF; $100 \mathrm{ng} / \mathrm{mL}$ of GDF5 or recombinant BMP2; neurobasal media with 6-10nM staurosporine or B27 supplement, $2 \mathrm{mM} \mathrm{L-glutamine} \mathrm{and} 10 \mu \mathrm{M}$ RA; $10 \mu \mathrm{M}$ RA and $5 \mu \mathrm{M}$ CAMP; $50 \mathrm{ng} / \mathrm{ml}$ GDNF; $10 \mu \mathrm{M}$ RA and 80nM tissue plasminogen activator 
Additional protocols used for differentiation may involve combinations of the above-mentioned methods, or a combination of $10 \mu \mathrm{M}$ RA and 0.3-5 mM dibutyryl cyclic adenosine monophosphate (dbcAMP) [39, 40], or of $10 \mu \mathrm{M}$ RA for 3 days and $80 \mathrm{nM}$ tissue plasminogen activator [41] or the protocol was not specified. Differentiation may also be caused by $200 \mathrm{ng} / \mathrm{mL}$ growth/differentiation factor 5 (GDF5) [42], recombinant bone morphogenetic protein 2 (BMP2) [42], staurosporine $[43,44]$ or $50 \mathrm{ng} / \mathrm{mL}$ glial cell line-derived neurotrophic factor (GDNF) [45]. The pros and cons of the differentiation of the SH-SY5Y cell line to obtain a relevant model for PD have been reviewed more extensively elsewhere [46]. Again, proper characterization of the differentiated cells is crucial. Next to the more conventional "ensemble" approaches, such as western blotting, transcriptomics or proteomics, the use of novel single-cell microscopy and single-cell RNAseq approaches will become instrumental to provide more detailed phenotypic profiling of the differentiated cell population [47, 48].

In view of the relevance of the DAergic system in PD, it is striking that among the papers using SH-SY5Y cells in PD research only 55 out of 962 examine the DAergic phenotype of the cell line (Table 2; for a list and details of the articles that checked the DAergic phenotype see Additional file 3). Remarkably, in some cases the DAergic phenotype has been studied but the results were not shown. The methods used to determine the SH-SY5Y DAergic phenotype include immunocytochemistry (ICC), western blot (WB), quantitative polymerase chain reaction (qPCR) and DA uptake/content. Of note, "ensemble" methods like WB and qPCR do not allow a distinction between expression changes in the whole population or in just a subset of cells. In this respect, ICC represents a more reliable technique to check the phenotype of the cell population. ICC with DAergic markers has been used in 26 out of the 56 articles: 10 in undifferentiated and 16 in differentiated cells (in general compared with undifferentiated controls). Comparison of all the provided ICC images, taking into account the cell source, media composition and differentiation method (when applicable), did not allow us to draw definitive conclusions about the phenotype of the undifferentiated cells, or about differences in outcome between various differentiation methods. Of note, no or only a few positive control images are shown in most articles, hindering the comparative literature survey. Also, SHSY5Y cells are known to respond inconsistently to the same differentiation treatment, depending on their cell source [49] or possibly passage number. Therefore, the variation reported may be at least in part due to the origin of the cells and different aspects regarding their handling, highlighting the importance of the proper reporting of all protocols involved.

\section{Mimicking PD}

In order to create SH-SY5Y-derived cell models that mimic PD, strategies are used based on drug treatment and/or genetic approaches with the manipulation of expression of candidate genes that have emerged from genetic studies in PD-families. Most papers (800 out of 962) choose one pharmacological or genetic strategy to force manifestation of a PD-like phenotype, but also multiple variants or combinations of these strategies have been used. The most-used compounds in drugbased approaches are 1-methyl-4-phenylpyridinium (MPP+), 6-hydroxydopamine (6-OHDA) and rotenone, which dysregulate multiple cellular pathways, focusing on mitochondrial dysfunction and oxidative stress (Table 3). MPP+ is the toxic metabolite of 1-methyl-4phenyl-1,2,3,6-tetrahydropyridine (MPTP), a by-product in the synthesis of 1-methyl-4-phenyl-4-propionoxy-piperidine (MPPP), a synthetic analog of heroin, which causes severe parkinsonism in humans when injected intravenously [50]. Since SH-SY5Y cells do not have the machinery to transform MPTP to MPP+, the metabolite itself is administered. Once inside the cell, MPP + inhibits complex I of the electron transport chain, impairing mitochondrial respiration and increasing reactive oxygen species (ROS) production, and redistributes DA to the cytosol, where it is oxidized and generates more ROS (reviewed in [51]). 6-OHDA is a catecholaminergic neurotoxin and its molecular mechanisms of action have been reviewed elsewhere [52]. Briefly, 6-OHDA enters

Table 3 Drug-based and genetic methods used to induce a PD-like phenotype in SH-SY5Y cells

\begin{tabular}{llll}
\hline PD-mimic & & & \\
\hline & Single & Multiple & Total \\
\hline MPP+ & 169 & 63 & 232 \\
$\begin{array}{l}\text { Manipulation of expression } \\
\text { of familial genes }\end{array}$ & 180 & 53 & 233 \\
6-OHDA & 141 & 47 & 188 \\
Rotenone & 69 & 56 & 125 \\
Dopamine & 33 & 26 & 59 \\
$\mathrm{H}_{2} \mathrm{O}_{2}$ & 12 & 34 & 46 \\
NM (R) Sal/Salsolinol & 21 & 6 & 27 \\
Paraquat & 13 & 12 & 25 \\
Lactacystin & 8 & 11 & 19 \\
Other treatments & & & 112
\end{tabular}

Listed are the most commonly used treatments to mimic PD in the SH-SY5Y cell line as well as the number of articles that use one (single) or, to validate the results, more than one (multiple) treatment. Other treatments include conditioned media from glial cells, MG132, SIN-1, staurosporine, thapsigargin, carbonyl cyanide $\mathrm{m}$ chlorophenyl hydrazine (CCCP), tunicamycin, epoxomicin, bafilomycin, neuromelanin, miRNAs, A- $\beta 1$, BmK1, L-buthionine-(S, R)-sulfoximine (BSO), Conduritol B epoxide (CBE), Ciplastin and PSI. MPP+: 1-methyl-4-phenylpyridinium; 6-OHDA: 6-hydroxydopamine; $\mathrm{H}_{2} \mathrm{O}_{2}$ : hydrogen peroxide 
catecholaminergic neurons via DA or NA transporters; it accumulates inside of the cell and triggers the formation of ROS and catecholamine quinones, leading to oxidative stress and cell death. Furthermore, 6-OHDA may inhibit complexes I and IV of the electron transport chain [53], although this has not been confirmed by others [54]. Exposure to rotenone, a highly lipophilic insecticide, has been linked to sporadic PD [55] and can directly enter cells, independently from transporters, to inhibit complex I, impairing mitochondrial respiration and enhancing ROS production, and inhibit proteasomal activity (reviewed in [56]).

Next to drug-based strategies also genetic approaches (e.g. knock down or forced overexpression of genes with mutations found in familial cases of PD) have been widely used to induce a PD-like phenotype (Table 3 ). Altogether 19 loci segregating with familial forms of PD are now known [57]. Of these only a subset have been used for reverse genetic manipulation, and overexpression of genetically encoded mutated variants of $\alpha$ synuclein (A30P, A53T, E46K, G51D, H50Q, S129A, S129D, S129E) or extracellularly added $\alpha$-synuclein variants is by far the most commonly used method to mimic PD in SH-SY5Y. Functional and rescue studies involving knockdown, overexpression or mutated forms of other genes, such as LRRK2 (G2019S, I2020T, R1441C, Y1699C), PINK1 (G309D, P209A, P399L, T313M), DJ-1 (A39S, C53A, C106A, L166P), ATP13A2, PLA2G6, GBA and Parkin (C289G, C431F, G328E, G430D, K161N, R42P, T240N, T240R, R265C, W453stop), have also been performed. Gene knockdown was mostly performed by transfection with siRNA or shRNA, while stable expression of mutated genes was achieved by their insertion into vectors such as pcDNA3.1 and transfection with reagents like Lipofectamine 2000 (ThermoFisher Scientific) or FuGENE * (Promega), adenoviral infection or lentiviral transduction. Surprisingly, novel revolutionary technologies such as the use of CRISPR/Cas9 or TALEN have not yet been applied in studies on PD, but we anticipate that reports on these tools for precision geneediting will appear soon. Collectively, the studies mimicking the genetic mutations present in PD have allowed the identification of a diverse set of pathways and molecular processes that are involved in the manifestation of the disease [58], including mitochondrial and mitophagy dysfunction $[59,60]$ and proteasomal and autophagy dysregulation, leading to protein aggregation [61-63]. One complementary strategy to study PD in cells is to interfere directly with one of these processes by administering specific compounds, with agonistic or antagonistic activity, such as hydrogen peroxide (oxidative stress), lactacystin/MG-123 (proteasome inhibitors), tunicamycin (N-glycosylation inhibitor, triggers ER stress), bafilomycin (inhibitor of vacuolar $\mathrm{H}^{+}$ATPase, leading to autophagy dysfunction), thapsigargin (inhibitor of the sarco/endoplasmic reticulum $\mathrm{Ca}^{2+}$ ATPase, resulting in ER stress and autophagy inhibition), carbonyl cyanide m-chlorophenyl hydrazone (CCCP) (inhibitor of oxidative phosphorylation, leading to mitochondrial dysfunction), Conduritol B epoxide (CBE) (GBA inhibitor), or salsolinol/staurosporine (cell death). Intriguingly, staurosporine, a broad-spectrum kinase inhibitor, has been used in some PD-related publications to induce cell death [64-72], while other publications have used it to induce DAergic differentiation and study PD-related features $[43,44]$. Early studies on SH-SY5Y cells showed differentiation towards a neuronal phenotype upon treatment with staurosporine [73, 74], which later has been characterized as catecholaminergic-like [75]. Therefore, the effects of different concentrations of staurosporine on SH-SY5Y cells should be characterized carefully to properly interpret studies that used this drug either as a differentiation agent or as an inducer of apoptosis. Figure 3 summarizes the now known cellular processes that are dysregulated in $\mathrm{PD}$, based on the analysis of the functions of the proteins encoded by the (mutated) familial PD genes, and the use of PDmimicking drugs in SH-SY5Y cells.

\section{Reproduction of PD-associated cellular phenotypes}

Apart from the DAergic phenotype, the ability of the cell line to reproduce the cellular abnormalities of PD is crucial for the validity of the model. One of the main hallmarks of PD is $\alpha$-synuclein aggregation [5]. To mimic this pathological feature, overexpression of WT $\alpha$-synuclein or stable expression of one of its familial mutations, such as A53T or A30P, has been successfully used [76-78]. Nevertheless, these manipulations do not always inevitably lead to increased formation of inclusions. Therefore, triggers such as cell differentiation together with $\mathrm{FeCl}_{2}$ (and $\mathrm{H}_{2} \mathrm{O}_{2}$ ) treatment, or Hsp70 blockage, are sometimes needed to observe $\alpha$-synuclein aggregation [79-82]. These different outcomes are possibly due to the specific $\alpha$-synuclein mutation used [77] or the level of expression achieved by the various constructs [78]. Interestingly, spontaneous $\alpha$-synuclein aggregation has been reported in non-transfected $\mathrm{SH}$ SY5Y cells [83]. Moreover, both differentiated and undifferentiated SH-SY5Y cells are sensitive to extracellular $\alpha$ synuclein-induced toxicity [84]. The cell line has also been used to study the kinetics and mechanisms of $\alpha$-synuclein degradation $[78,85]$, the link between $\alpha$ synuclein aggregation and intracellular calcium [86], and other pathological changes of $\alpha$-synuclein, such as its carboxyl-terminal cleavage [32]. Other PD-related problems, such as abnormal mitochondrial function, oxidative stress and autophagy or proteasomal dysfunction, have been reproduced in SH-SY5Y cells as well. These 


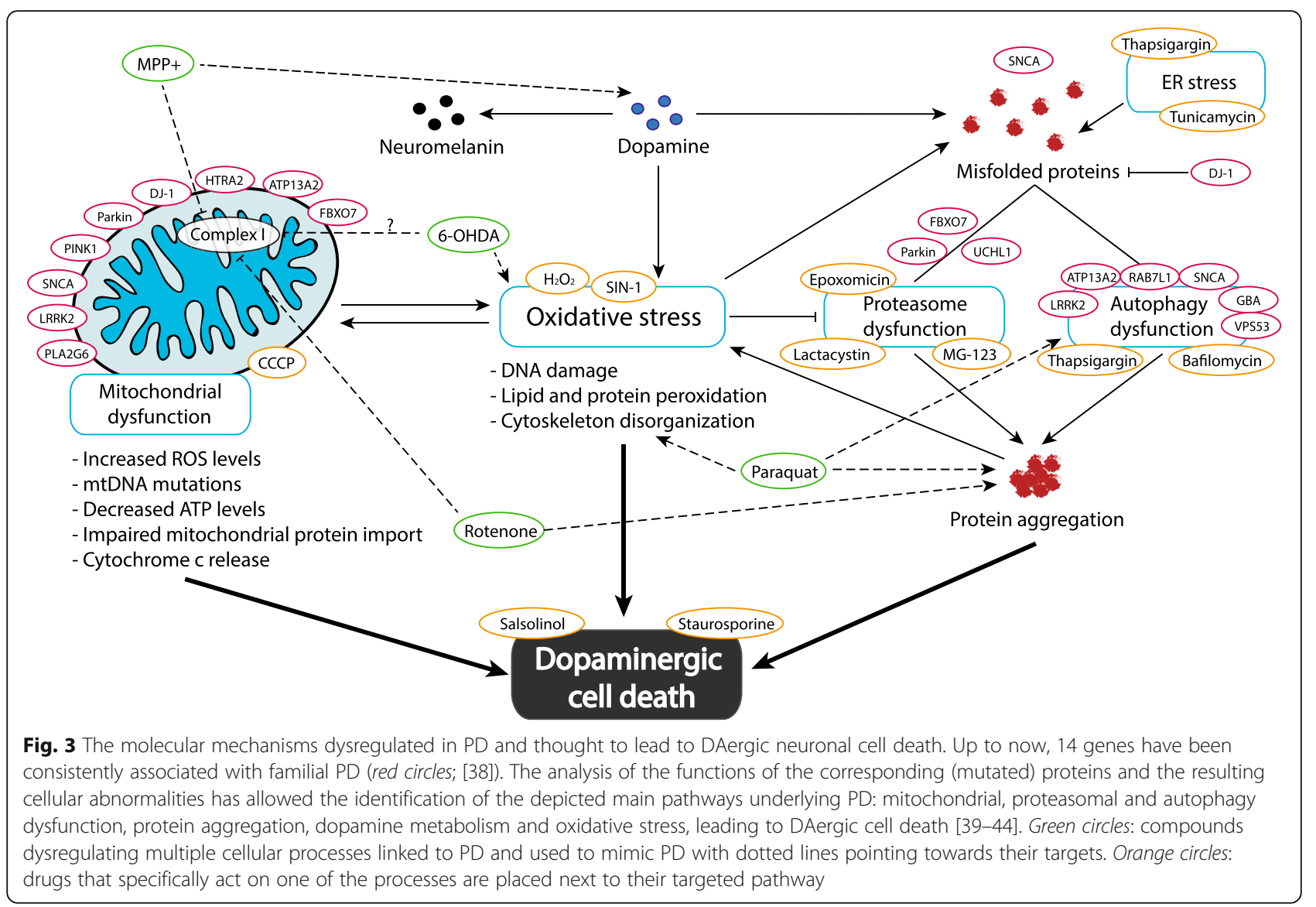

hallmarks are usually triggered by the administration of specific drugs (Fig. 3) or, alternatively, by the knockdown of a gene corresponding to a familial PD-gene or expression of a familial PD-gene. For example, silencing of PINK1 leads to mitochondrial dysfunction $[87,88]$ and mutated forms of $\alpha$-synuclein impair proteasomal activity [89]. The additive effects that are caused by coexpression of familial PD genes can also be observed in this cell line (e.g. cotransfection of $\alpha$-synuclein and LRRK2 enhances the formation of aggregates, phosphorylation, cell-to-cell transmission and extracellular release of $\alpha$-synuclein [90]). Therefore, SH-SY5Y cells represent an attractive tool to study most cellular alterations linked to PD and strategies to ameliorate their effects, but a careful experimental setting is required when analyzing $\alpha$-synuclein aggregation, since the findings vary between studies. Yet, not all aspects of PD pathobiology can be faithfully studied in SH-SY5Y cells, and therefore investigations into for example electrophysiological abnormalities or neurochemical dysfunction require other and more complex models, such as primary dopaminergic cultures or brain slices, ex vivo.
In vitro and in vivo models used in parallel with SH-SY5Y cells

PD is characterized by the loss of DAergic neurons from the SN [5]. Thus, primary cultures of neurons from this brain area of patients and controls may be considered the most reliable models to unravel the molecular mechanisms underlying this disease. However, the inaccessibility and lack of proliferation of such neurons largely precludes their use. Conversely, as discussed above, the use of a proliferative and more uniform model like the SH-SY5Y cell model has also limitations. Therefore in many studies other cell types have been employed in parallel. Of the articles analyzed, $67.6 \%$ reported experiments performed exclusively in the SH-SY5Y cell line and $19.7 \%$ used in parallel other cell lines with a neuronal phenotype, including rodent mesencephalic primary cultures and (mainly cortical) primary neurons, stem cells, PC12 cell line, Neuro-2a cell line or MN9D cell line (Table 4). The remaining $12.7 \%$ used cell lines that are not neuronal (-like), such as HEK293, HeLa or glial cells. Primary cell cultures are physiologically more relevant than immortalized cell lines, but they are difficult 
Table 4 Use of alternative cellular models in parallel to SH-SY5Y cells

\begin{tabular}{ll}
\hline Alternative cellular models & \#articles \\
\hline No other neuronal cell lines & 772 \\
Other neuronal (-like) cell lines & 190 \\
Mesencephalic cultures (mouse/rat) & 54 \\
Primary neurons (cortical mainly) (mouse/rat) & 54 \\
PC12 (rat) & 39 \\
Neuro-2a (mouse) & 13 \\
hESC, NPSC, hMSC, iPSCs (human) & 9 \\
SK-N-BE (2)-M17(M17) (human) & 9 \\
MN9D (mouse) & 7 \\
Other & 45
\end{tabular}

The table specifies the number of articles (\#articles) that do not use any neuronal cell line other than SH-SY5Y cells; and those that do use another neuronal (-like) cell line (and the most commonly used ones). Sometimes an article uses multiple alternative cell lines and, thus, the addition of the individual values of other neuronal (-like) cell lines is larger than the number of articles that use other neuronal (-like) cell lines (190). More detailed information on these other cell lines can be found in Additional file 4

to maintain and, depending on the age of the source animals or the dissection accuracy, can introduce experimental variability [91]. Since the SN is located in the mesencephalon, or midbrain, rodent mesencephalic primary cultures are enriched for the cell population of interest, the SN DAergic neurons. Primary cultures from other brain regions (mainly cortex) have also been extensively used as complementary in vitro models, but these cultures consist of mixed populations of various neuronal subtypes. Furthermore, primary cultures of DAergic neurons from other species, such as the worm C. elegans, have been used [92, 93]. In general, the overtly less physiologically relevant permanently established cell lines are easier to maintain than cells in primary cultures (because the cell lines are generally rendered immortal) and can sometimes be differentiated into terminal neuronal populations. The PC12 cell line $\left(\right.$ ATCC $^{\circ}$ CRL-1721 $^{\mathrm{m}}$ ) has been derived from a rat pheochromocytoma and widely used to study PD. This lineage has a chromaffin-like character, and shares its embryonic origin with DAergic neurons. Nerve growth factor treatment of $\mathrm{PC} 12$ cells induces differentiation into a catecholaminergic-like phenotype [94, 95]. The Neuro-2a cell line (ATCC ${ }^{\circ}$ CCL- $131^{\text {mom }}$ ) has been derived from a mouse brain neuroblastoma and can be differentiated into neuronal-like cells [96] and, more specifically, to DAergic neurons by a dbcAMP treatment [97]. Other PD cellular models include cell hybrids, such as the MN9D cell line [98], and derivatives from the neuroblastoma SK cell line. A full list of neuronal (-like) cell models used in parallel with SH-SY5Y cells can be found in Additional file 4. It is important to note that most of the alternative lineages are derived from species other than human. Finally, patient-derived induced pluripotent stem cells (iPSCs) that can be differentiated into DAergic neurons (and possibly other relevant cell types such as glial cells) represent an emerging new category of cell models for PD [99-101].

Apart from cellular models, about $21 \%$ of the articles that use SH-SY5Y cells also employ an animal model for PD to validate and further understand the cellular results. The animal models include mouse, rat, C. elegans and fruit fly and the PD features in these in vivo models are mimicked with MPTP, 6-OHDA, rotenone or genetic mutations (reviewed in $[102,103]$ ).

\section{Conclusions}

This systematic review illustrates the "popularity" and broad use of the neuroblastoma cell line SH-SY5Y in PD research and underlines some of its drawbacks. SHSY5Y cells have been used to study the molecular and cellular mechanisms underlying the effects of some of the PD-related toxins, to perform functional studies on familial PD genes, and to test putative protective compounds for PD treatment. Thus, this cell line has been a valuable asset to help unravel the molecular complexity of PD. However, SH-SY5Y cells are not purely DAergic because the cell line was obtained as a neuroblastoma derivative and thus has cancerous properties that influence its differentiation fate, viability, growth performance, metabolic properties and genomic stability. Hence, SH-SY5Y cells possess physiological characteristics which differ greatly from the normal DAergic neuronal features. Reports on the exact SH-SY5Y phenotype are contradictory. Differences in cell source and maintenance in culture, perhaps of epigenetic character, could explain these variations, but the lack of accurate reporting of experimental protocol parameters and inaccurate listing of individual characteristics of cell lineages kept at different laboratories hinders the drawing of firm conclusions. Therefore, the cell source has to be specifically indicated and more studies on the effects of media composition on the cell population are needed to compare findings, and catalyze reproducibility and progress with this PD-model. In addition, the use of other neuronal (-like) cell lines, such as those reviewed here, and animal models in parallel with SH-SY5Y cells may help to validate the findings. The choice of these additional models should take into account aspects such as species differences, tumorigenic properties and time and resource requirements. A further topic regarding the use of SH-SY5Y cells concerns the differentiation regime that - until now - has been used to drive the cell line towards a DAergic phenotype. Variations in the outcome of the differentiation protocol could again be due to the origin and handling of the cells. Furthermore, the use of chemical compounds to differentiate the cell line into a 
more DAergic or neuronal population may affect parameters that are not directly linked to the desired phenotype, and they may produce confounding effects. The systematic use of ICC and other single cell assays, together with qPCR and WB to characterize the phenotype of the entire cell population, is required for a proper validation of the DAergic phenotype of the SH-SY5Y cells as a disease model. The method of choice to model PD is crucial especially because the onset of this multifactorial disease involves both genetic and environmental factors. Genetic as well as chemical approaches have been used in the functional studies on SH-SY5Y cells to target one or multiple pathways linked to PD. In any case, the use of multiple approaches in parallel is recommended and expected to be facilitated by current developments in the fields of chemical biology and reverse-genetics (i.e. CRISPR/Cas9 applications) that will allow a much broader application of chemical libraries for cellsignaling inhibition and genome editing, respectively. These novel opportunities together with the proper exploitation of the already well-established procedures for cell culturing will allow the standardization of the use of the SH-SY5Y cell line and maximize the benefit from this appealing cell model for PD.

\section{Additional files}

Additional file 1: List of articles resulting from the literature search and article inclusion/exclusion in this review. Complete list of articles that were obtained with the search terms "Parkinson", "Parkinson's", or "Parkinson's disease" and "neuroblastoma", "SH-SY5Y", "SHSY5Y, or "SHSY$5 Y^{\prime \prime}$ in PubMed until the $22^{\text {nd }}$ of November 2016. The inclusion and exclusion criteria are specified. (XLSX $263 \mathrm{~kb}$ )

Additional file 2: Compositions of the media used for culturing $\mathrm{SH}$ SY5Y cells. The table displays the composition of the media used in each of the articles included in the review. (XLSX 55 kb)

Additional file 3: Techniques and markers used to determine the DAergic phenotype of SH-SY5Y cells. List of articles in which the DAergic phenotype of the cell line was experimentally validated, and the techniques and markers that were used, together with the cell source, media used and differentiation included or not. (XLSX 18 kb)

Additional file 4: Alternative neuronal (-like) cell lines used to validate the findings in SH-SY5Y cells. The table contains the neuronal (-like) cell lines that have been used in parallel with SH-SY5Y cells to study PD and the species from which they are derived, the cell type and the number of articles that used the cell line. (XLSX $13 \mathrm{~kb}$ )

\section{Abbreviations}

6-OHDA: 6-hydroxydopamine; ATCC: American Type Culture Collection; BDNF: Brain-derived neurotrophic factor; DA: Dopamine;

DAergic: Dopaminergic; DSMZ: German collection of Microorganisms and Cell Cultures; ECACC: European Collection of Authenticated Cell Cultures; ICC: Immunocytochemistry; MPP+: 1-methyl-4-phenylpyridinium; MPTP: 1-methyl-4-phenyl-1,2,3,6-tetrahydropyridine; NA: Noradrenaline; PD: Parkinson's disease; qPCR: Quantitative polymerase chain reaction; RA: Retinoic acid; ROS: Reactive oxygen species; SN: Substantia nigra; TPA: 12-O-Tetradecanoylphorbol-13-acetate; WB: Western blot

Acknowledgements

Not applicable.
Funding

HX was funded by a Ph.D. grant from Radboudumc.

Availability of data and materials

All data generated and analyzed during this study is included in this published article and its additional files.

\section{Authors' contributions}

HX compiled the information and wrote the manuscript. GJMM and BW supervised and revised the writing of the manuscript. All authors approved the final version of the manuscript.

\section{Competing interests}

The authors declare that they have no competing interests.

\section{Consent for publication}

Not applicable.

Ethics approval and consent to participate

Not applicable.

\section{Author details}

'Department of Cell Biology, Radboud Institute for Molecular Life Sciences (RIMLS), Radboudumc, Nijmegen, The Netherlands. ${ }^{2}$ Department of Molecular Animal Physiology, Donders Institute for Brain, Cognition and Behaviour, Radboud University, Nijmegen, The Netherlands.

Received: 4 October 2016 Accepted: 5 January 2017

Published online: 24 January 2017

\section{References}

1. Dorsey ER, Constantinescu R, Thompson JP, Biglan KM, Holloway RG, Kieburtz K, et al. Projected number of people with Parkinson disease in the most populous nations, 2005 through 2030. Neurology. 2007;68(5):384-6.

2. Wirdefeldt K, Adami H-O, Cole P, Trichopoulos D, Mandel J. Epidemiology and etiology of Parkinson's disease: a review of the evidence. Eur J Epidemiol. 2011;26 Suppl 1:1-58.

3. Chaudhuri KR, Schapira AH. Non-motor symptoms of Parkinson's disease: dopaminergic pathophysiology and treatment. Lancet Neurol. 2009;8(5):464-74.

4. Xia R, Mao Z-H. Progression of motor symptoms in Parkinson's disease. Neurosci Bull. 2012;28(1):39-48.

5. Dexter DT, Jenner P. Parkinson disease: from pathology to molecular disease mechanisms. Free Radic Biol Med. 2013;62:132-44.

6. Biedler JL, Roffler-Tarlov S, Schachner M, Freedman LS. Multiple Neurotransmitter Synthesis by Human Neuroblastoma Cell Lines and Clones. Cancer Res. 1978;38(11 Part 1):3751-7.

7. Påhlman S, Ruusala A-I, Abrahamsson L, Mattsson MEK, Esscher T. Retinoic acid-induced differentiation of cultured human neuroblastoma cells: a comparison with phorbolester-induced differentiation. Cell Differ. 1984; 14(2):135-44.

8. Ross RA, Biedler JL. Presence and Regulation of Tyrosinase Activity in Human Neuroblastoma Cell Variants in Vitro. Cancer Res. 1985;45(4):1628-32

9. Nagatsu T, Levitt M, Udenfriend S. Tyrosine hydroxylase. The initial step in norepinephrine synthesis. J Biol Chem. 1964;239:2910-7.

10. Levin EY, Levenberg B, Kaufman S. The enzymatic conversion of 3,4-dihydroxyphenylethylamine to norepinephrine. J Biol Chem. 1960:235:2080-6.

11. Krishna A, Biryukov M, Trefois C, Antony PMA, Hussong R, Lin J, et al. Systems genomics evaluation of the SH-SY5Y neuroblastoma cell line as a model for Parkinson's disease. BMC Genomics. 2014;15:1154.

12. Wu X, Lin M, Li Y, Zhao X, Yan F. Effects of DMEM and RPMI 1640 on the biological behavior of dog periosteum-derived cells. Cytotechnology. 2009; 59(2):103-11.

13. Huang Z, Shao W, Gu J, Hu X, Shi Y, Xu W, Huang C, Lin D. Effects of culture media on metabolic profiling of the human gastric cancer cell line SGC7901. Mol Biosyst. 2015;11(7):1832-40.

14. Carey BW, Finley LW, Cross JR, Allis CD, Thompson CB. Intracellular a-ketoglutarate maintains the pluripotency of embryonic stem cells. Nature. 2015;518(7539):413-6. 
15. Mazzio E, Soliman KF. Pyruvic acid cytoprotection against 1-methyl-4phenylpyridinium, 6-hydroxydopamine and hydrogen peroxide toxicities in vitro. Neurosci Lett. 2003;337(2):77-80.

16. Wang X, Perez E, Liu R, Yan LJ, Mallet RT, Yang SH. Pyruvate protects mitochondria from oxidative stress in human neuroblastoma SK-N-SH cells. Brain Res. 2007;1132(1):1-9.

17. van der Valk J, Brunner D, De Smet K, Fex Svenningsen A, Honegger $P$, Knudsen LE, Lindl T, Noraberg J, Price A, Scarino ML, Gstraunthaler G. Optimization of chemically defined cell culture media-replacing fetal bovine serum in mammalian in vitro methods. Toxicol In Vitro. 2010; 24(4):1053-63.

18. Buttiglione M, Vitiello F, Sardella E, Petrone L, Nardulli M, Favia P, et al. Behaviour of SH-SY5Y neuroblastoma cell line grown in different media and on different chemically modified substrates. Biomaterials. 2007;28(19):2932-45.

19. Agholme L, Lindström T, Kågedal K, Marcusson J, Hallbeck M. An In Vitro Model for Neuroscience: Differentiation of SH-SY5Y Cells into Cells with Morphological and Biochemical Characteristics of Mature Neurons. Alzheimer's Dis. 2010;20(4):1069-82.

20. Kovalevich J, Langford D. Considerations for the Use of SH-SY5Y Neuroblastoma Cells in Neurobiology. 2013. p. 9-21.

21. Encinas M, Iglesias M, Liu Y, Wang H, Muhaisen A, Ceña V, et al. Sequential Treatment of SH-SY5Y Cells with Retinoic Acid and Brain-Derived Neurotrophic Factor Gives Rise to Fully Differentiated, Neurotrophic FactorDependent, Human Neuron-Like Cells. J Neurochem. 2002;75(3):991-1003.

22. Sarkanen J-R, Nykky J, Siikanen J, Selinummi J, Ylikomi T, Jalonen TO. Cholesterol supports the retinoic acid-induced synaptic vesicle formation in differentiating human SH-SY5Y neuroblastoma cells. J Neurochem. 2007; 102(6):1941-52.

23. Schneider L, Giordano S, Zelickson BR, Johnson SM, Benavides AG, Ouyang $X$, Fineberg N, Darley-Usmar VM, Zhang J. Differentiation of SH-SY5Y cells to a neuronal phenotype changes cellular bioenergetics and the response to oxidative stress. Free Radic Biol Med. 2011;51(11):2007.

24. Teppola H, Sarkanen J-R, Jalonen TO, Linne M-L. Morphological Differentiation Towards Neuronal Phenotype of SH-SY5Y Neuroblastoma Cells by Estradiol, Retinoic Acid and Cholesterol. Neurochem Res. 2016:41(4):731-47.

25. Yang H, Wang J, Sun J, Liu X, Duan W-M, Qu T. A new method to effectively and rapidly generate neurons from SH-SY5Y cells. Neurosci Lett. 2016;610:43-7.

26. Lopes FM, Schröder R, da Júnior MLC F, Zanotto-Filho A, Müller CB, Pires AS, et al. Comparison between proliferative and neuron-like SH-SY5Y cells as an in vitro model for Parkinson disease studies. Brain Res. 2010;1337:85-94.

27. Cheung Y-T, Lau WK-W, Yu M-S, Lai CS-W, Yeung S-C, So K-F, et al. Effects of all-trans-retinoic acid on human SH-SY5Y neuroblastoma as in vitro model in neurotoxicity research. Neurotoxicology. 2009;30(1):127-35.

28. Korecka JA, van Kesteren RE, Blaas E, Spitzer SO, Kamstra JH, Smit AB, Swaab DF, Verhaagen J, Bossers K. Phenotypic Characterization of Retinoic Acid Differentiated SH-SY5Y Cells by Transcriptional Profiling. PLoS One. 2013:8(5):e63862.

29. Hashemi SH, Li J-Y, Ahlman H, Dahlström A. SSR2(a) receptor expression and adrenergic/cholinergic characteristics in differentiated SH-SY5Y cells. Neurochem Res. 2003;28(3-4):449-60.

30. Cheng B, Martinez AA, Morado J, Scofield V, Roberts JL, Maffi SK. Retinoic acid protects against proteasome inhibition associated cell death in $\mathrm{SH}$ SY5Y cells via the AKT pathway. Neurochem Int. 2013:62(1):31-42.

31. Presgraves SP, Borwege S, Millan MJ, Joyce JN. Involvement of dopamine D2/D3 receptors and BDNF in the neuroprotective effects of S32504 and pramipexole against 1-methyl-4-phenylpyridinium in terminally differentiated SH-SY5Y cells. Exp Neurol. 2004;190(1):157-70.

32. Bellucci A, Collo G, Sarnico I, Battistin L, Missale C, Spano P. Alpha-synuclein aggregation and cell death triggered by energy deprivation and dopamine overload are counteracted by D 2 D 3 receptor activation. J Neurochem. 2008;106(2):560-77.

33. Knaryan VH, Samantaray S, Park S, Azuma M, Inoue J, Banik NL. SNJ-1945, a calpain inhibitor, protects SH-SY5Y cells against MPP (+) and rotenone. J Neurochem. 2014;130(2):280-90.

34. Grimm J, Mueller A, Hefti F, Rosenthal A. Molecular basis for catecholaminergic neuron diversity. Proc Natl Acad Sci U S A. 2004;101(38):13891-6.

35. Poulin J-F, Zou J, Drouin-Ouellet J, Kim K-YA, Cicchetti F, Awatramani RB. Defining midbrain dopaminergic neuron diversity by single-cell gene expression profiling. Cell Rep. 2014;9(3):930-43.
36. Edsjö A, Lavenius E, Nilsson H, Hoehner JC, Simonsson P, Culp LA Martinsson T, Larsson C, Påhlman S. Expression of trkB in human neuroblastoma in relation to MYCN expression and retinoic acid treatment. Lab Invest. 2003:83(6):813-23.

37. Goldie BJ, Barnett MM, Cairns MJ. BDNF and the maturation of posttranscriptional regulatory networks in human SH-SY5Y neuroblast differentiation. Front Cell Neurosci. 2014;8:325.

38. Mastroeni D, Grover A, Leonard B, Joyce JN, Coleman PD, Kozik B, Bellinger $\mathrm{DL}$, Rogers J. Microglial responses to dopamine in a cell culture model of Parkinson's disease. Neurobiol Aging. 2009;30(11):1805-17.

39. Morton AJ, Williams MN, Emson PC, Faull RL. The morphology of human neuroblastoma cell grafts in the kainic acid-lesioned basal ganglia of the rat. J Neurocytol. 1995;24(8):568-84.

40. Nisar R, Hanson PS, He L, Taylor RW, Blain PG, Morris CM. Diquat causes caspase-independent cell death in SH-SY5Y cells by production of ROS independently of mitochondria. Arch Toxicol. 2015;89(10):1811-25.

41. Su C, Yang X, Lou J. Geniposide reduces a-synuclein by blocking microRNA21/lysosome-associated membrane protein 2A interaction in Parkinson disease models. Brain Res. 2016;1644:98-106.

42. Hegarty SV, Sullivan AM, O'Keeffe GW. BMP2 and GDF5 induce neuronal differentiation through a Smad dependant pathway in a model of human midbrain dopaminergic neurons. Mol Cell Neurosci. 2013;56:263-71.

43. Borland MK, Trimmer PA, Rubinstein JD, Keeney PM, Mohanakumar K, Liu L, Bennett Jr JP. Chronic, low-dose rotenone reproduces Lewy neurites found in early stages of Parkinson's disease, reduces mitochondrial movement and slowly kills differentiated SH-SY5Y neural cells. Mol Neurodegener. 2008;3:21

44. Ballaz S, Morales I, Rodríguez M, Obeso JA. Ascorbate prevents cell death from prolonged exposure to glutamate in an in vitro model of human dopaminergic neurons. J Neurosci Res. 2013;91(12):1609-17.

45. Wang T, Chen J, Tang CX, Zhou XY, Gao DS. Inverse Expression Levels of EphrinA3 and EphrinA5 Contribute to Dopaminergic Differentiation of Human SH-SY5Y Cells. J Mol Neurosci. 2016:59(4):483-92.

46. Xie H, Hu L, Li G. SH-SY5Y human neuroblastoma cell line: in vitro cell model of dopaminergic neurons in Parkinson's disease. Chin Med J (Engl). 2010;123(8):1086-92.

47. Wu AR, Neff NF, Kalisky T, Dalerba P, Treutlein B, Rothenberg ME, Mburu FM, Mantalas GL, Sim S, Clarke MF, Quake SR. Quantitative assessment of singlecell RNA-sequencing methods. Nat Methods. 2014;11(1):41-6.

48. Battich N, Stoeger T, Pelkmans L. Control of Transcript Variability in Single Mammalian Cells. Cell. 2015;163(7):1596-610.

49. Wang H, Imai Y, Kataoka A, Takahashi R. Cell Type-Specific Upregulation of Parkin in Response to ER Stress. Antioxid Redox Signal. 2007;9(5):533-42.

50. Langston J, Ballard P, Tetrud J, Irwin I. Chronic Parkinsonism in humans due to a product of meperidine-analog synthesis. Science. 1983; 219(4587):249-54.

51. Vila M, Przedborski S. Neurological diseases: Targeting programmed cell death in neurodegenerative diseases. Nat Rev Neurosci. 2003;4(5):365-75.

52. Simola N, Morelli M, Carta AR. The 6-hydroxydopamine model of Parkinson's disease. Neurotox Res. 2007;11(3-4):151-67.

53. Glinka YY, Youdim MBH. Inhibition of mitochondrial complexes I and IV by 6-hydroxydopamine. Eur J Pharmacol Environ Toxicol Pharmacol. 1995; 292(3-4):329-32.

54. Storch A, Kaftan A, Burkhardt K, Schwarz J. 6-Hydroxydopamine toxicity towards human SH-SY5Y dopaminergic neuroblastoma cells: independent of mitochondrial energy metabolism. J Neural Transm. 2000;107(3):0281-93.

55. Tanner CM, Kamel F, Ross GW, Hoppin JA, Goldman SM, Korell M, et al. Rotenone, paraquat, and Parkinson's disease. Environ Health Perspect. 2011; 119(6):866-72.

56. Cicchetti F, Drouin-Ouellet J, Gross RE. Environmental toxins and Parkinson's disease: what have we learned from pesticide-induced animal models? Trends Pharmacol Sci. 2009;30(9):475-83.

57. Kumaran R, Cookson MR. Pathways to Parkinsonism Redux: convergent pathobiological mechanisms in genetics of Parkinson's disease. Hum Mol Genet. 2015:24(R1):R32-44.

58. Michel PP, Hirsch EC, Hunot S. Understanding Dopaminergic Cell Death Pathways in Parkinson Disease. Neuron. 2016;90(4):675-91.

59. Exner N, Lutz AK, Haass C, Winklhofer KF. Mitochondrial dysfunction in Parkinson's disease: molecular mechanisms and pathophysiological consequences. EMBO J. 2012;31(14):3038-62. 
60. Ryan BJ, Hoek S, Fon EA, Wade-Martins R. Mitochondrial dysfunction and mitophagy in Parkinson's: from familial to sporadic disease. Trends Biochem Sci. 2015;40(4):200-10.

61. McNaught KSP, Olanow CW. Protein aggregation in the pathogenesis of familial and sporadic Parkinson's disease. Neurobiol Aging. 2006;27(4):530-45.

62. Dehay B, Martinez-Vicente M, Caldwell GA, Caldwell KA, Yue Z, Cookson MR, et al. Lysosomal impairment in Parkinson's disease. Mov Disord. 2013;28(6):725-32.

63. Zhang $H$, Duan $C$, Yang $H$. Defective Autophagy in Parkinson's Disease: Lessons from Genetics. Mol Neurobiol. 2015;51(1):89-104.

64. Kahns S, Lykkebo S, Jakobsen LD, Nielsen MS, Jensen PH. Caspase-mediated parkin cleavage in apoptotic cell death. J Biol Chem. 2002;277(18):15303-8.

65. Muqit MM, Davidson SM, Payne Smith MD, MacCormac LP, Kahns S, Jensen $\mathrm{PH}$, Wood NW, Latchman DS. Parkin is recruited into aggresomes in a stress-specific manner: over-expression of parkin reduces aggresome formation but can be dissociated from parkin's effect on neuronal survival. Hum Mol Genet. 2004;13(1):117-35.

66. Petit A, Kawarai T, Paitel E, Sanjo N, Maj M, Scheid M, Chen F, Gu Y, Hasegawa H, Salehi-Rad S, Wang L, Rogaeva E, Fraser P, Robinson B, St George-Hyslop P, Tandon A. Wild-type PINK1 prevents basal and induced neuronal apoptosis, a protective effect abrogated by Parkinson diseaserelated mutations. J Biol Chem. 2005;280(40):34025-32.

67. Alves da Costa C, Dunys J, Brau F, Wilk S, Cappai R, Checler F. 6Hydroxydopamine but not 1-methyl-4-phenylpyridinium abolishes alphasynuclein anti-apoptotic phenotype by inhibiting its proteasomal degradation and by promoting its aggregation. J Biol Chem. 2006;281(14):9824-31.

68. Iwashita A, Muramatsu Y, Yamazaki T, Muramoto M, Kita Y, Yamazaki S, Mihara K, Moriguchi A, Matsuoka N. Neuroprotective efficacy of the peroxisome proliferator-activated receptor delta-selective agonists in vitro and in vivo. J Pharmacol Exp Ther. 2007;309(3):1067-78.

69. Ruan Q, Harrington AJ, Caldwell KA, Caldwell GA, Standaert DG. VPS41, a protein involved in lysosomal trafficking, is protective in Caenorhabditis elegans and mammalian cellular models of Parkinson's disease. Neurobiol Dis. 2010;37(2):330-8

70. Jaworska-Feil L, Jantas D, Leskiewicz M, Budziszewska B, Kubera M, Basta-Kaim A, Lipkowski AW, Lason W. Protective effects of TRH and its analogues against various cytotoxic agents in retinoic acid (RA)differentiated human neuroblastoma SH-SY5Y cells. Neuropeptides. 2010:44(6):495-508

71. Kawajiri S, Machida Y, Saiki S, Sato S, Hattori N. Zonisamide reduces cell death in SH-SY5Y cells via an anti-apoptotic effect and by upregulating MnSOD. Neurosci Lett. 2010;481(2):88-91.

72. Verhaar R, Drukarch B, Bol JG, Jongenelen CA, Musters RJ, Wilhelmus MM. Increase in endoplasmic reticulum-associated tissue transglutaminase and enzymatic activation in a cellular model of Parkinson's disease. Neurobiol Dis. 2012;58(7):785-93.

73. Shea TB, Beermann ML. Staurosporine-induced morphological differentiation of human neuroblastoma cells. Cell Biol Int Rep. 1991;15(2):161-8.

74. Jalava A, Heikkila J, Minnamaija L, Akerman K, Pahlman S. Staurosporine induces a neuronal phenotype in Sh-SY5Y human neuroblastoma cells that resembles that induced by the phorbol ester 12-0-tetradecanoyl phorbol-13 acetate (TPA). FEBS. 1992;300(2):114-8.

75. Filograna R, Civiero L, Ferrari V, Codolo G, Greggio E, Bubacco L, et al. Analysis of the Catecholaminergic Phenotype in Human SH-SY5Y and BE (2)-M17 Neuroblastoma Cell Lines upon Differentiation. PLoS ONE. 2015; 10(8):e0136769.

76. Tofaris GK, Layfield R, Spillantini MG. alpha-synuclein metabolism and aggregation is linked to ubiquitin-independent degradation by the proteasome. FEBS Lett. 2001;509(1):22-6.

77. Pandey N, Schmidt RE, Galvin JE. The alpha-synuclein mutation E46K promotes aggregation in cultured cells. Exp Neurol. 2006;197(2):515-20.

78. Liangliang $X$, Yonghui $H$, Shunmei $E$, Shoufang $G$, Wei Z, Jiangying Z. Dominant-positive HSF1 decreases alpha-synuclein level and alphasynuclein-induced toxicity. Mol Biol Rep. 2010;37(4):1875-81.

79. Hasegawa T, Matsuzaki M, Takeda A, Kikuchi A, Akita H, Perry G, Smith MA Itoyama Y. Accelerated alpha-synuclein aggregation after differentiation of SH-SY5Y neuroblastoma cells. Brain Res. 2004;1013(1):51-9.

80. Zhang Z, Cheng Y. miR-16-1 promotes the aberrant a-synuclein accumulation in parkinson disease via targeting heat shock protein 70. ScientificWorldJournal. 2014;2014:938348.

81. Macchi F, Deleersnijder A, Van den Haute C, Munck S, Pottel H, Michiels A, Debyser Z, Gerard M, Baekelandt V. High-content analysis of a-synuclein aggregation and cell death in a cellular model of Parkinson's disease. J Neurosci Methods. 2016:261:117-27.

82. Izumi Y, Kondo N, Takahashi R, Akaike A, Kume T. Reduction of Immunoreactivity Against the C-Terminal Region of the Intracellular a-Synuclein by Exogenous a-Synuclein Aggregates: Possibility of Conformational Changes. J Parkinsons Dis. 2016;6(3):569-79.

83. Xin W, Emadi S, Williams S, Liu Q, Schulz P, He P, Alam NB, Wu J, Sierks MR. Toxic Oligomeric Alpha-Synuclein Variants Present in Human Parkinson's Disease Brains Are Differentially Generated in Mammalian Cell Models. Biomolecules. 2015;5(3):1634-51.

84. Emadi S, Kasturirangan S, Wang MS, Schulz P, Sierks MR. Detecting morphologically distinct oligomeric forms of alpha-synuclein. J Biol Chem. 2009;284(17):11048-58.

85. Bennett MC, Bishop JF, Leng Y, Chock PB, Chase TN, Mouradian MM. Degradation of alpha-synuclein by proteasome. J Biol Chem. 1999; 274(48):33855-8.

86. Follett J, Darlow B, Wong MB, Goodwin J, Pountney DL. Potassium depolarization and raised calcium induces a-synuclein aggregates. Neurotox Res. 2013:23(4):378-92

87. Gegg ME, Cooper JM, Schapira AH, Taanman JW. Silencing of PINK1 expression affects mitochondrial DNA and oxidative phosphorylation in dopaminergic cells. PLoS One. 2009:4(3):e4756.

88. van der Merwe C, van Dyk HC, Engelbrecht L, van der Westhuizen FH, Kinnear C, Loos B6, Bardien S. Curcumin Rescues a PINK1 Knock Down SHSY5Y Cellular Model of Parkinson's Disease from Mitochondrial Dysfunction and Cell Death. Mol Neurobiol. 2016.

89. Nonaka T, Hasegawa M. A cellular model to monitor proteasome dysfunction by alpha-synuclein. Biochemistry. 2009;48(33):8014-22.

90. Kondo K, Obitsu S, Teshima R. a-Synuclein aggregation and transmission are enhanced by leucine-rich repeat kinase 2 in human neuroblastoma SH-SY5Y cells. Biol Pharm Bull. 2011;34(7):1078-83.

91. Al-Ali H, Blackmore M, Bixby JL, Lemmon VP. High Content Screening with Primary Neurons. 2014

92. Shi Z, Lu Z, Zhao Y, Wang Y, Zhao-Wilson X, Guan P, et al. Neuroprotective effects of aqueous extracts of Uncaria tomentosa: Insights from 6-OHDA induced cell damage and transgenic Caenorhabditis elegans model. Neurochem Int. 2013:62(7):940-7.

93. Johnson WM, Golczak M, Choe K, Currran PL, Miller OG, Yao C, et al. Regulation of DJ-1 by glutaredoxin 1 in vivo - implications for Parkinson's disease. Biochemistry. 2016;55(32):4519-32.

94. Greene LA, Tischler AS. Establishment of a noradrenergic clonal line of rat adrenal pheochromocytoma cells which respond to nerve growth factor. Proc Natl Acad Sci U S A. 1976;73(7):2424.

95. Westerink RHS, Ewing AG. The PC12 cell as model for neurosecretion. Acta Physiol (Oxf). 2008;192(2):273.

96. Klebe RJ, Ruddle FH. Neuroblastoma: cell culture analysis of a differentiating stem cell system. J Cell Biol. 1969;43:69a.

97. Tremblay RG, Sikorska M, Sandhu JK, Lanthier P, Ribecco-Lutkiewicz M, BaniYaghoub M. Differentiation of mouse Neuro 2A cells into dopamine neurons. J Neurosci Methods. 2010;186(1):60-7.

98. Choi HK, Won LA, Kontur PJ, Hammond DN, Fox AP, Wainer BH, et al. Immortalization of embryonic mesencephalic dopaminergic neurons by somatic cell fusion. Brain Res. 1991;552(1):67-76.

99. Soldner F, Hockemeyer D, Beard C, Gao Q, Bell GW, Cook EG, et al. Parkinson's disease patient-derived induced pluripotent stem cells free of viral reprogramming factors. Cell. 2009;136(5):964-77.

100. Hartfield EM, Fernandes HJR, Vowles J, Cowley SA, Wade-Martins R, Liu X, et al. Cellular reprogramming: a new approach to modelling Parkinson's disease. Biochem Soc Trans. 2012;40(5):1152-7.

101. Badger JL, Cordero-Llana O, Hartfield EM, Wade-Martins R. Parkinson's disease in a dish - Using stem cells as a molecular tool. Neuropharmacology. 2014;76:88-96.

102. Blesa J, Przedborski S. Parkinson's disease: animal models and dopaminergic cell vulnerability. Front Neuroanat. 2014;8:155.

103. Jagmag SA, Tripathi N, Shukla SD, Maiti S, Khurana S. Evaluation of Models of Parkinson's Disease. Front Neurosci. 2015:9:503. 